\title{
Concepciones de la escritura y de la alfabetización académica en devoluciones escritas de docentes universitarios: un estudio contrastivo
}

\author{
Márcia Sipavicius Seide* \\ Lucía Antonia Natale**
}

\begin{abstract}
Resumo
En este artículo, presentamos un estudio contrastivo, de carácter exploratorio, sobre las concepciones de la escritura y de la alfabetización académica que se construyen y reproducen en los comentarios escritos de dos profesoras universitarias. A partir de la selección de dos casos elegidos intencionalmente, nos enfocamos en el examen de los aspectos que se constituyen en objeto de atención en los comentarios de dos docentes, una de Brasil, que dicta una asignatura inicial y otra de Argentina, a cargo de una materia de final de carrera. Estos datos son complementados con entrevistas semiestructuradas a las profesoras que participaron del estudio. El análisis contempla los distintos discursos de la escritura sistematizados por Ivanič (2004) y los modelos de alfabetización caracterizados por Lea y Street (1998). Los resultados muestran que, según el análisis de los comentarios, podría pensarse que, más allá de algunas coincidencias, las concepciones de escritura y de alfabetización académica de las docentes son distintas. Sin embargo, la puesta en relación de esos datos con los recolectados en las entrevistas evidencia visiones comunes. Asimismo, se encontró que las diferencias en los resultados iniciales se correlacionan con los objetivos de las asignaturas y con distintas necesidades de los estudiantes.

Palabras clave: Escritura. Alfabetización académica. Devoluciones escritas. Concepciones. Docentes universitarios.
\end{abstract}




\section{Introducción}

Desde las últimas décadas del siglo XX, se ha puesto de relieve la necesidad de iniciar a los estudiantes universitarios en la producción escrita de géneros ${ }^{1}$ propios de las culturas disciplinares (HYLAND, 2004) en las que desean insertarse. En ese marco, se han realizado importantes investigaciones sobre los procesos de enseñanza y aprendizaje de los discursos de las disciplinas. Se han estudiado los dispositivos y estrategias (cursos, talleres y centros de escritura, por ejemplo) adoptados por las universidades para facilitar la alfabetización académica ${ }^{2}$ (BAZERMAN et al. 2005; CARLINO, 2003; 2013); los géneros solicitados a los estudiantes (NESI; GARDNER, 2012; PARODI, 2008); las consignas de los docentes (NATALE, 2013); los rasgos genéricos (SWALES, 1990; 2004; NESI; GARDNER, 2012), entre otras temáticas relevantes. En este artículo nos concentramos en las devoluciones escritas y comentarios que los docentes universitarios realizan sobre las producciones de sus alumnos. Ese tipo particular de interacción constituye una importante vía para la enseñanza de la escritura (HYLAND; HYLAND, 2001), aunque no ha recibido la suficiente atención en estudios del campo.

Las investigaciones realizadas sobre el tema, principalmente en países anglosajones, indagaron acerca de los resultados educativos, como la efectividad de la corrección de errores, las preferencias de los alumnos sobre el tipo de retroalimentación y sus valoraciones acerca de las devoluciones de los docentes (para un exhaustivo estado del arte, véase HYLAND; HYLAND, 2006a).

1 La noción de género admite distintas definiciones en distintas corrientes de investigación y enseñanza de la alfabetización académica. Las líneas anglosajonas que constituyen la base de nuestro estudio, la Nueva Retórica, la Escuela de Sídney y el Inglés para propósitos específicos (véase Hyon, 1996), si bien guardan algunas diferencias de énfasis, comparten con la noción bajtiniana la interrelación entre uso del lenguaje en contexto y actividad social en su definición.

2 Existen en español distintos términos para designar a los procesos de enseñanza y aprendizaje de la lectura y la escritura. El más extendido es el de "Alfabetización". Derivado de la traducción del término inglés Literacy se refiere a un proceso continuo de construcción de conocimientos sobre prácticas lingüísticas "que se co-construye socialmente en el continuo de la evolución individual del ser humano" (BRASLAVSKY, 2004, p.70). En esta misma línea, Paula Carlino entiende la alfabetización académica como "el conjunto de nociones y estrategias necesarias para participar en la cultura discursiva de las disciplinas, así como en las actividades de producción y análisis de textos requeridas para aprender en la universidad" (CARLINO, 2003, p. 410). En un trabajo posterior, Carlino enfatiza el rol que adquieren en este proceso "las acciones que han de realizar los profesores, con apoyo institucional, para que los alumnos universitarios aprendan a exponer, argumentar, resumir, buscar información, jerarquizarla, ponerla en relación, valorar razonamientos, debatir, etcétera, según los modos típicos de hacerlo en cada materia". (CARLINO, 2013, p. 371). 
También se ha estudiado el foco de atención de los comentarios y se observó que, tradicionalmente, se atendió a cuestiones lingüísticas pero con el tiempo, se privilegió el contenido conceptual (COFFIN et al., 2003; FERRIS, 2003). Desde una perspectiva discursiva, algunas investigaciones analizaron las funciones que asumen los comentarios de los docentes y el tipo de relaciones que se establecen entre estudiantes y docentes (HYLAND; HYLAND, 2001; 2006a, NATALE, 2013c). Otros estudios (ANSON, 1989; IVANIČ et al., 2000; JEFFERY; SELTING, 1999) examinaron las representaciones sobre la escritura y el conocimiento que se construyen en las devoluciones escritas. Se encontró que los comentarios de los docentes constituyen "mensajes" acerca de ellos mismos y de las instituciones en que se desempeñan, así como las ideologías predominantes en su contexto, las que se verán reproducidas y reforzadas en las devoluciones escritas.

En este artículo, llevamos adelante un estudio exploratorio sobre aspectos que no han sido aún suficientemente indagados. Nos interesa examinar cuáles son las concepciones de la escritura y de la alfabetización académica que se construyen y se reproducen en los comentarios escritos de los profesores universitarios y si es posible observar el modo peculiar de producir conocimiento de determinada asignatura, como señalan Lea y Street (1998). En efecto, los autores llaman la atención sobre "the relationship between feedback and epistemological issues of knowledge construction" (LEA; STREET, 1998, p. 168) y se preguntan:

How is feedback being used to direct students to develop and write their academic knowledge in very specific ways within particular courses which are implicitly presented as 'common-sense ways of knowing'? (LEA; STREET, 1998, p. 168).

Específicamente, nos planteamos como interrogantes los siguientes: ¿Pueden rastrearse en las devoluciones el área de conocimiento de la asignatura, sus objetivos generales y su ubicación en el plan de estudios? ¿Se vinculan esos datos externos con el foco de atención del comentario? ¿Existe una relación entre ese foco con el modelo de alfabetización académica delineado?

Para responder estos interrogantes, nos valemos de la caracterización de los "discursos sobre la escritura"3 establecidos por Ivanič (2004) y en los distintos

3 Ivanič define "discursos sobre la escritura y el aprendizaje de la escritura" como "sets of beliefs about writing and learning to write, and practices of teaching and assessment of writing associated with these beliefs" (IVANIČ, 2004, p. 220-221). Es decir que la noción se asocia con un conjunto de creencias asociadas entre sí que pueden ser rastreadas, según señala la propia autora (IVANIČ, 2004, p. 220), tanto en fuentes lingüísticas (documentos, entrevistas, materiales pedagógicos) como en prácticas de 
modelos de alfabetización académica caracterizados por Lea y Street (1998), aunque nos parece importante asentar que, tal como han señalado los autores, pueden encontrarse superposiciones entre los distintos discursos y modelos, de manera que los más abarcadores incluyen también los que atienden a los aspectos más básicos. En cuanto a la metodología, adoptamos una perspectiva contrastiva, con un corpus acotado. A partir de la selección de dos casos elegidos intencionalmente, nos enfocamos en el examen de los aspectos que se constituyen en objeto de atención en los comentarios de dos docentes (una de Brasil y otra de Argentina). Siguiendo a Martin (1992) y en relación con los planteos de Ivanič (2004), distinguimos como estratos a los que se dirigen los comentarios los siguientes: genérico, discursivo, léxico-gramatical y notacional.

El artículo se organiza de la siguiente manera. En primer lugar, exponemos los distintos discursos sobre la escritura descriptos por Ivanič (2004), que establecen un claro diálogo con los distintos modelos de alfabetización académica consignados por Lea y Street (1998). A continuación, se presentan los casos, que se analizan en dos instancias. La primera se concentra en los comentarios y en la segunda, se la complementa con los datos recolectados en entrevistas semiestructuradas a las docentes. Para finalizar, discutimos los resultados y sus implicancias pedagógicas para programas institucionales de alfabetización académica.

\section{Enseñanza de la escritura en distintos encuadres para la alfabetización académica}

Según Ivanič (2004), las prácticas de enseñanza de la escritura y los respectivos discursos que las sustentan forman parte de complejos sistemas de creencias sobre la escritura y el lenguaje, organizados por estratos que van de lo más interno - el nivel del texto - hasta los más externos- el nivel sociocultural, en el que están implicados la ideología y las relaciones de poder que se juegan en una comunidad discursiva. Esos diferentes modos de concebir la escritura constituyen seis configuraciones discursivas: 1) discurso de las habilidades; 2) discurso de la creatividad; 3) discurso del proceso; 4) discurso del género; 5) discurso de la práctica social; 6) discurso sociopolítico (IVANIČ, 2004, p.225). 
En el primero, la escritura es asociada con una serie de conocimientos sobre normas y patrones sintácticos aplicables a todos los textos, por lo que importa considerar las palabras, las oraciones y los lazos cohesivos necesarios para articular oraciones (IVANIČ, 2004). El texto aparece como un producto autónomo, una organización coherente de elementos estructurados de acuerdo con un sistema de reglas que puede funcionar independientemente de los contextos (HYLAND, 2002). Para la enseñanza, se propone un entrenamiento explícito para el dominio de la gramática y reglas prescriptivas (HYLAND, 2002; IVANIČ, 2004). El docente aparece como un experto que instruye a los novatos, quienes son representados en un rol meramente pasivo, como receptor de las normas establecidas (HYLAND, 2002). En relación con la alfabetización académica, ese discurso se encuentra en los programas de numerosos cursos ubicados a inicios de los estudios de grado, en los que se entrena a los estudiantes para el manejo de fórmulas gramaticales y convenciones generales del discurso científico-académico (LEA; STREET, 1998; CARLINO, 2013).

En el discurso que considera la escritura como una manifestación de la creatividad, se valora, especialmente, la expresión del autor, el contenido y, sobre todo, el estilo (HYLAND, 2002; IVANIČ, 2004). Se considera que se aprende a escribir escribiendo, a partir de tópicos interesantes e inspiradores. Esa perspectiva acarrea una noción de aprendizaje de tipo natural. El docente es un facilitador que pone a la disposición de los estudiantes materiales de lectura atractivos. Los alumnos emergen como individuos activos, en interacción con el entorno letrado (HYLAND, 2002).

Los discursos de la escritura como proceso son el resultado de diversas investigaciones enmarcadas en psicología cognitiva (FLOWER; HAYES, 1981; SCARDAMALIA; BEREITER, 1987). Centrados en la figura del escritor (HYLAND, 2002), esos modelos conciben la escritura como un proceso de carácter recursivo, no lineal, que comprende tanto actividades mentales, como otras de ejecución práctica. El aprendizaje de la escritura incluye la adquisición de una serie de procedimientos para componer un texto. El docente asume la figura de facilitador de la tarea, no da directivas, sino que asiste y coopera con los estudiantes. Hasta hoy, ese paradigma es adoptado por profesores que consideran importante evaluar distintas versiones de un texto.

El discurso, que asocia la escritura con un conjunto de géneros o tipos textuales moldeados por el contexto social, muestra una visión más amplia que 
las perspectivas anteriores. Si bien los textos constituyen un objeto de análisis privilegiado, no son ya considerados como entidades autónomas; son relacionados con los eventos sociales en que se originan y con los propósitos que persiguen. En ese enfoque, aprender a escribir es aprender las características lingüísticas de los géneros para reproducirlos, apropiadamente, en determinados contextos con el fin de lograr ciertas metas (IVANIČ, 2004). Si bien la adquisición de los géneros puede llevarse adelante de manera implícita, por la participación en el medio social -como sostiene Bajtín (1979) respecto de los primarios- se entiende que la enseñanza explícita es una mejor opción (HYLAND, 2002; 2003). El docente guía a los estudiantes en los pasos de la composición estructural de los géneros; es un sujeto activo, atento a los avances de los estudiantes para proponer nuevos desafíos.

En algunas corrientes también basadas en géneros, el énfasis no recae tanto en la dimensión textual, sino en el contexto en que se desarrollan las actividades sociales que les dan origen. La escritura aparece así como una práctica social (IVANIČ, 2004). La alfabetización adquiere en ese discurso un sentido más abarcador, que no solo comprende la escritura, sino también un conjunto de prácticas sociales. La alfabetización, entonces, no se ve restringida a los ámbitos educativos, sino que abarca todas las esferas de la vida social, como se propone desde la perspectiva de los Nuevos Estudios de la Alfabetización (STREET, 1984). Esas propuestas resultan por cierto enriquecedoras, ya que no limitan las experiencias de los aprendices al ámbito educativo y destacan la importancia de la participación de los sujetos en actividades sociales. Sin embargo, los lineamientos para la enseñanza no se han desarrollado suficientemente aún (IVANIČ, 2004; LILLIS, 2003).

En el discurso que concibe la escritura como una práctica sociocultural se sostiene que la escritura y el lenguaje, en general, son moldeados por las fuerzas sociales y las relaciones de poder. En ese sentido, el escritor no es un sujeto completamente libre en sus elecciones lingüísticas, sino que es influido por el contexto sociopolítico. Por otra parte, la escritura contribuye con la configuración de su identidad (IVANIČ, 1998). A partir de esos postulados, se hace hincapié en la necesidad de que los estudiantes desarrollen una conciencia crítica sobre los discursos y los géneros que emplean y se propone una enseñanza de tipo explícito, que incluya: a) explicaciones sobre los factores sociopolíticos relacionados con su uso, b) la reflexión sobre la manera en que las elecciones lingüísticas posicionan 
a lectores y escritores y c) la indagación sobre las opciones disponibles para introducir cambios en la representación de la realidad social (IVANIČ, 2004, p. 238).

De acuerdo con Ivanič (2004), a su vez, esos discursos pueden superponerse en el quehacer docente concreto y un programa completo sobre la escritura académica puede abarcar todos ellos.

Como ya señalamos anteriormente, la categorización propuesta por Ivanič (2004) se relaciona con los modelos de alfabetización académica propuestos por Lea y Street (1998), quienes encuentran tres tipos de abordaje de las alfabetizaciones (LEA; STREET, 1998): el de habilidades de estudio, el de socialización académica y el de las alfabetizaciones académicas, de los cuales, los dos primeros son los más frecuentemente adoptados. El de habilidades de estudio presupone una visión de la escritura como un fenómeno básicamente cognitivo e individual. Se focaliza en aspectos gramaticales y léxicos, considerándose que pueden ser transferidas a cualquier contexto. Se liga, así, con el discurso de la escritura como un conjunto de habilidades, aunque incluye la dimensión procesual cuando se enfatiza la redacción de textos. En el modelo de socialización académica, se privilegia el proceso de enculturación de los estudiantes en las distintas disciplinas, a través de las prácticas de lectura y escritura de los géneros más habituales, los que se reconocen como estables y no son puestos en cuestión.

Esos dos planteos de la alfabetización descriptos son caracterizados como monológicos, en tanto las prácticas pedagógicas e institucionales promueven la reproducción de los discursos oficiales de las disciplinas (LILLIS, 2003, p. 193-195). Por ello, se propone un tercer modelo, llamado "alfabetizaciones académicas", que no solo se preocupa por los lenguajes y los conocimientos de las distintas disciplinas, sino, también, por la epistemología y las relaciones de poder y autoridad establecidas dentro de cada comunidad, así como por la construcción de la identidad de los escritores. En este modelo predominan los discursos sobre la escritura como una práctica social y cultural, marcada por procesos sociopolíticos. Para Lea y Street $(1998,2006)$, los tres modelos de alfabetización no son mutuamente excluyentes, sino que el tercero incluye a los anteriores, aunque desde una posición crítica, reflejada en la adopción del plural para designar la diversidad de prácticas que se llevan adelante en las instituciones (LILLIS; SCOTT, 2007). 


\section{Los estudios de caso}

Teniendo como punto de partida los modelos de alfabetización reconocidos por Lea y Street (1998) y el sistema de concepciones y creencias sobre la escritura propuesto por Ivanič (2004), seleccionamos y delineamos los dos casos de estudio que se presentan y analizan a continuación, para lo que seguimos un enfoque contrastivo. Cotejamos las devoluciones escritas realizadas por dos profesoras, una brasileña y una argentina, a las que, respectivamente, llamaremos con los nombres ficticios de María y Mariana. Para responder a nuestros interrogantes, consideramos, principalmente, sus comentarios pero sumamos datos obtenidos en entrevistas semiestructuradas. Los resultados obtenidos, a su vez, generaron reflexiones pedagógicas y educativas.

Ambas docentes dictan cursos de grado, aunque en distintas carreras y en distintos momentos. María dicta una materia inicial de la carrera de Letras en una universidad pública de Paraná, Brasil y Mariana, una destinada a estudiantes de la licenciatura en Política Social próximos a su graduación en una institución también pública ubicada en los suburbios de la capital de Argentina. Ambas tienen un firme interés en ocuparse de la enseñanza de la escritura en sus cursos, lo que se verifica en solicitar trabajos escritos que son comentados por las docentes para que los estudiantes puedan presentar versiones mejoradas. Así, se encuentra que ambas profesoras hacen un seguimiento de las producciones escritas de los estudiantes.

María dicta la asignatura de Historia de la Lengua Portuguesa, ofertada en el primer año de una licenciatura en Letras Lengua Portuguesa y una lengua extranjera -alemana, española o inglesa. En la entrevista, hecha un semestre después de impartido el curso, María señaló que, en relación con la enseñanza de escritura académica, tenía como objetivo principal transformar su materia en una asignatura de escritura intensiva y promover una primera experiencia de alfabetización académica en los estudiantes.

Para alcanzar ese objetivo, decidió solicitarles, como principal dispositivo evaluativo, dos versiones de un mismo texto y cooperar con ellos en el proceso de redacción para obtener mejores resultados. En la instancia de evaluación final, solicitó la escritura de un artículo. El alumno pudo elegir un tópico entre cuatro propuestos por la profesora, los cuales habían sido abordados en clase a partir de la lectura de artículos científicos. Se esperaba que en este trabajo se utilizara 
información del texto analizado en clase además de otros textos elegidos por los alumnos. El escrito podía ser hecho individualmente o en grupos de hasta cuatro personas. Se escribieron 13 trabajos. Los estudiantes produjeron una primera versión que enviaron por e-mail a la profesora. La docente añadió varios comentarios al margen de los textos. Dos semanas después, tuvieron lugar encuentros presenciales con los grupos, en los cuales los comentarios fueron explicados y complementados oralmente. Una semana después de la reunión, cada grupo le envió a la profesora la versión final del texto.

Con respecto a Mariana, al momento de ser entrevistada, colaboraba con el dictado de "Planificación y Gestión de la Política Social II", una asignatura avanzada, orientada al desempeño profesional de los estudiantes. Las tareas escritas solicitadas anticipan las que los estudiantes realizarán en su ejercicio profesional: deben investigar la implementación de un programa social de salud en una localidad con el objetivo de elaborar una evaluación sobre su ejecución. Periódicamente, a lo largo del semestre, los alumnos entregan distintos informes de avance, que son comentados por las docentes con procesadores de texto de uso corriente.

En la entrevista, Mariana se refirió, también, a los estudiantes y a sus procesos de aprendizaje de la escritura disciplinar. Señaló que, a su juicio, las dificultades de los estudiantes no se deben a su origen sociocultural (como suele escucharse en muchas instituciones), sino que radicarían en una falta de "entrenamiento" y de “preparación”, tarea que es, según ella, responsabilidad de los docentes, por lo que la asume. La metáfora del entrenamiento, a la que Mariana apela para representar el proceso de enseñanza, se recupera en las devoluciones escritas a partir de las instrucciones de la docente y apuntan a una concepción de la escritura como proceso, la misma utilizada por María cuando afirma que delineó su asignatura de modo a que se volviera una asignatura de escritura intensiva.

\section{$4 \mathrm{El}$ análisis de los comentarios}

Para el análisis de los comentarios de las docentes, se seleccionó una devolución escrita de cada profesora. Se eligió el texto que hubiera recibido la mayor cantidad de comentarios realizados por las docentes, los cuales fueron analizados siguiendo los estratos propuestos por Martin (1992): género, discurso, léxico-gramatical y gráfico. 
En el nivel de género, consideramos las anotaciones referidas a los propósitos del texto, las partes o pasos que componen el escrito y, también, la adecuación a la situación comunicativa, la consideración de los participantes del intercambio y la adopción de un registro acorde. En el plano discursivo, incluimos las anotaciones que llaman la atención sobre la organización coherente de los contenidos, el avance de la información en el texto, la construcción de párrafos y sus interconexiones. El estrato léxico-gramatical contempla la construcción de cláusulas y oraciones y la selección del léxico propio de la disciplina. Finalmente, en el nivel notacional, se incluyen tanto la ortografía como la construcción de las referencias bibliográficas que, si bien son en un punto mecánicas, guardan estrecha relación con el plano del contexto y constituyen uno de los aspectos que los estudiantes deben aprender en los inicios de la vida académica.

Los resultados se muestran en la Tabla 1 que presenta, inclusive, ejemplos de comentarios de ambas profesoras.

Tabla 1: Ejemplos de comentarios realizados por María y Mariana según los estratos considerados.

\begin{tabular}{|l|l|l|}
\hline Estrato & Ejemplos ${ }^{1}$ María & Ejemplos Mariana \\
\hline \multirow{5}{*}{ Genérico } & $\begin{array}{l}\text { (1) En relación al texto como } \\
\text { un todo: muitas seções } \\
\text { estão herméticas, difíceis de } \\
\text { entender; há bastante plágio; } \\
\text { as informações oriundas } \\
\text { do livro de fontes podem } \\
\text { ser melhor aproveitadas; } \\
\text { será preciso escrever com } \\
\text { as próprias palavras e } \\
\text { reformular o projeto de } \\
\text { artigo deixando-o mais curto. }\end{array}$ & $\begin{array}{l}\text { (1) En relación a la introducción: } \\
\text { Armar un sub apartado que } \\
\text { principales conceptos a trabajar } \\
\text { sobre la base de APS Revisar } \\
\text { problema y preguntas de } \\
\text { investigación articulando con los } \\
\text { objetivos grales (i?) generales ¿? } \\
\text { y específicos de la parte cuanti y } \\
\text { cuali. }\end{array}$ \\
\hline \multirow{5}{*}{ Discursivo } \\
& $\begin{array}{l}\text { (3) aqui vcs apenas } \\
\text { informam que o sistema } \\
\text { vocálico do quadro 08 foi } \\
\text { analisado como não válido } \\
\text { para a língua portuguesa... } \\
\text { mas por quem o foi? Como } \\
\text { esta avaliação foi feita? }\end{array}$ & $\begin{array}{l}\text { interpretativo, incluso marcando } \\
\text { algunas diferencias interesantes o } \\
\text { no... y por qué creen que sucede } \\
\text { lo que sucede }\end{array}$ \\
\hline
\end{tabular}




\begin{tabular}{|l|l|l|}
\hline $\begin{array}{l}\text { Léxico } \\
\text { gramatical }\end{array}$ & $\begin{array}{l}\text { (5) Nesta frase vocês usaram } \\
\text { a conjunção "todavia", } \\
\text { não seria melhor usar a } \\
\text { conjunção "ainda"? }\end{array}$ & $\begin{array}{l}\text { (6) Redacción, eviten frases tan } \\
\text { largas; impiden la comprensión. }\end{array}$ \\
\hline Notacional & $\begin{array}{l}\text { (7) Informar o número de } \\
\text { página, se não consultou a } \\
\text { obra, colocar apud }\end{array}$ & $\begin{array}{l}\text { (8) Nunca vi citar de esta forma. } \\
\text { Poner acentos. }\end{array}$ \\
\hline
\end{tabular}

Fonte: Elaborada por las autoras.

Antes de analizar la distribución de los porcentajes de comentarios dirigidos a cada uno de los estratos considerados para el análisis (Tabla 2), consideramos necesario explicitar los criterios mediante los cuales incluimos los ejemplos anteriores en cada uno de los estratos. Como puede observarse, los comentarios clasificados como correspondientes al género se vinculan, por un lado, con la organización de los textos en secciones y, por el otro, con las normas propias de los círculos académicos, que los géneros acarrean. En el ejemplo (1), no solo se llama la atención sobre las partes del texto, sino, también, sobre la obligación de evitar el plagio y reformular las fuentes para dar cuenta de una visión propia.

En (2), el acento está puesto en la adecuación de las secciones a los modelos canónicos del género y se demanda en un rasgo esencial del proyecto de investigación: la coherencia interna entre problema, preguntas y objetivos. En otras palabras, la docente dirige sus comentarios a propiciar un texto que se ajuste a las convenciones genéricas.

Con respecto al estrato discursivo, encontramos que las profesoras plantean preguntas que solicitan el desarrollo de la información de modo que los estudiantes dejen de manifiesto sus análisis e interpretaciones, como se observa en los ejemplos (3) y (4). Indudablemente, las validaciones e interpretaciones que se reclaman no pueden ser desgajadas del nivel genérico, ya que forman parte de cualquier tipo de texto que circule en el medio académico. Sin embargo, los comentarios no hacen referencia a las secciones o pasos ni a la adecuación al registro, sino que están planteados en términos del avance de la información y de las interconexiones entre los contenidos desarrollados.

En cuanto a (5) y (6), los hemos clasificado como ejemplos de comentarios referidos al estrato léxico-gramatical porque se focalizan sobre aspectos puntuales de la construcción de las cláusulas: el uso de un conector y la construcción de las 
frases, respectivamente.

Por último, de acuerdo con los ejemplos referidos a la notación - (7) y (8) - las dos profesoras manifiestan su interés por el aprendizaje de las normas de citación, lo que está relacionado con el género y las reglas propias del ámbito académico, cuestión que retomamos más adelante.

En la Tabla 2, se muestran los porcentajes de comentarios referidos a cada estrato:

Tabla 2: estratos considerados en los comentarios de María y Mariana, en porcentajes.

\begin{tabular}{|l|l|l|}
\hline Estrato & $\begin{array}{l}\text { Comentarios de } \\
\text { María, en porcentajes }\end{array}$ & $\begin{array}{l}\text { Comentarios de Mariana, en } \\
\text { porcentajes }\end{array}$ \\
\hline Genérico & 18,45 & 35,0 \\
\hline Discursivo & 8,00 & 41,7 \\
\hline Léxico gramatical & 1,96 & 10,0 \\
\hline Notacional & 70,58 & 13,3 \\
\hline Total & 100 & 100 \\
\hline
\end{tabular}

Fonte: Elaborada por las autoras.

Las cifras de la Tabla 2 muestran que ambas profesoras hacen comentarios que contemplan todos los estratos, los que se encuentran en distintas versiones de un mismo trabajo, revelándonos que las dos comparten una concepción de la escritura como proceso y una concepción de aprendizaje basada en las necesidades de los alumnos, como veremos más adelante. Sin embargo, también encontramos algunas diferencias, según el foco de atención de los comentarios de cada docente.

En los comentarios de María, predominan las anotaciones dirigidas al estrato notacional, seguido del genérico. Esa constatación podría sugerir que María adhiere a una visión de la escritura puramente normativa y que su visión de la alfabetización se limita al de desarrollo de habilidades, especialmente, las de citación, ya que la mayoría de esos comentarios apuntan a la cuestión de las normas para construir referencias bibliográficas. Pero ese dato no puede ser leído de manera aislada ya que, si bien se refiere a una cuestión de normativa, se relaciona, directamente, con la búsqueda del dominio de reglas del ámbito académico. En todo caso, la cifra obtenida se vincula con el hecho de que los estudiantes, por estar iniciando 
sus carreras, no han logrado aún incorporar tales normas. Desde otra perspectiva, sigue en orden la cantidad de comentarios referidos al estrato genérico, aunque en un porcentaje relativamente bajo, si se lo compara con las cifras de Mariana. Una posible explicación es que los alumnos tuvieron pocos desaciertos en cuanto a la organización del texto solicitado debido a las sucesivas lecturas de artículos de revistas realizadas a lo largo del curso.

Por otro lado, los datos de Mariana evidencian que sus sugerencias se dirigen, principalmente, a los estratos genérico y discursivo. El primero, como sabemos, se relaciona con las prácticas discursivas propias de la comunidad disciplinar. En los ejemplos relevados, se nota una preocupación por la producción de conocimientos, comunicados en un informe de investigación, lo que se observa en las referencias a "objetivos", "hipótesis", "problemas", "apartados", "criterios teóricos", por ejemplo. Así, las observaciones de Mariana se vinculan con los objetivos de la asignatura, que procura que los estudiantes aprendan a evaluar programas sociales a partir de un análisis planteado como una investigación. Esa actividad requiere de la formulación de un problema, interrogantes, hipótesis e indagación empírica, con la consiguiente creación de nuevos saberes.

Por otra parte, en cuanto al nivel discursivo, que es el que mayor cantidad de comentarios ha generado, se observa que las sugerencias de la docente apuntan a un mayor grado de explicitación y de precisión en la información que sustenta las afirmaciones de los estudiantes, lo que está se asocia con la dimensión genérica y con el contexto en que circula el escrito.

En efecto, uno de los requisitos de un texto académico o profesional es el desarrollo de los argumentos que sostienen una aseveración. Así, también podemos afirmar que muchos de los comentarios de Mariana apuntan al desarrollo de una visión propia de los problemas por parte de los estudiantes y, consecuentemente, se proponen vigorizar su voz autoral. En ese sentido, si consideramos esas observaciones, puede pensarse que Mariana apunta a una visión de la escritura que atiende, especialmente, a los géneros y las prácticas socioculturales, por lo que estaría enmarcada en un modelo de alfabetización que procura la socialización académica.

Así planteado el análisis, podría pensarse que las docentes adhieren a modelos de alfabetización muy diferentes, sin embargo, creemos que la sola observación de los comentarios puede arrojar una lectura sesgada y que, por tanto, resulta necesario complementar este examen con otros datos. 


\section{Los objetivos pedagógicos}

Vistas las diferencias en los estratos prioritariamente considerados por las docentes, pasaremos a observar qué nos dicen otros datos, de modo de complejizar la mirada y obtener un panorama más amplio. Así, para responder nuestras preguntas, tuvimos, también en cuenta, los datos recolectados en las entrevistas. En ellas, las docentes manifestaron sus preocupaciones sobre el aprendizaje de los alumnos, sus objetivos con respecto la enseñanza de la escritura académica y algunas de las estrategias de enseñanza adoptadas. De acuerdo con ello, encontramos en ambas otro aspecto en común, además de una perspectiva procesual de la escritura: la consideración de las necesidades de los alumnos, aunque cada una de las profesoras partió de distintos puntos. María reencaminó su quehacer docente cuando observó, empíricamente, las necesidades de los estudiantes con respecto a la alfabetización académica. Mariana, en cambio, partió de lo que, según su experiencia previa, requieren los alumnos para participar en círculos profesionales: un entrenamiento sistemático para la escritura.

Además, las entrevistas nos permitieron reconocer otros factores intervinientes en las decisiones docentes. De acuerdo con las informaciones que nos brindó María, a lo largo del curso, se ocupó de atender a todos los estratos y contempló distintas dimensiones y concepciones sobre la escritura, con excepción de la creativa. Para atender al género y la socialización académica, en varias de las clases expositivas, se detuvo en la lectura de un conjunto de artículos específicos de la disciplina tomados de una misma revista científica. Ese tipo de lectura focalizada en el género implica presentar a los alumnos textos avalados por una comunidad discursiva concreta: la de los investigadores de lingüística histórica del Grupo de Estudios Lingüísticos del Estado de São Paulo - GEL.

La lectura analizada en clase, entre otros aspectos, sirvió para examinar en detalle la estructura y las características discursivas de un conjunto de artículos escritos por expertos en los contenidos de la asignatura. La profesora se focalizó así en una visión que presenta la escritura como una práctica social y disciplinar. Por otro lado, algunas veces, se hicieron observaciones sobre el ethos (la construcción del autor como una autoridad en el área) de quien escribe artículos y las características concretas del género que permiten relevar las relaciones de poder en una comunidad. 
De acuerdo con lo relatado por María, la actividad antes mencionada se vincula con los propósitos pedagógicos perseguidos en relación con la escritura. Esos consistían en promover, a través del trabajo final, un primer evento de alfabetización académica. Ella instó a los alumnos a buscar, elegir, estudiar y parafrasear investigaciones publicadas sobre unos de los tópicos de la asignatura. Se esperaba que ellos lograsen no sólo interpretar y organizar información de modo coherente y sintético, sino que se interesaran por buscar más información sobre el tema elegido de forma autónoma y crítica. Si bien forman parte del proceso de alfabetización académica, no se exigió, en esa instancia, la producción de conocimiento nuevo, la aplicación de la teoría a nuevos datos o a nuevas situaciones, la reflexión sobre metodologías o cuestiones sobre la relación entre práctica y teoría y sus potencialidades frente a la resolución de problemas.

El conocimiento de esas informaciones recolectadas en la entrevista nos permite, entonces, contextualizar los comentarios en un marco más amplio, relativo a la planificación global de la asignatura, los propósitos y las actividades. Así, el tipo y el foco de los comentarios parecen consistentes con los objetivos planteados para la enseñanza de la escritura (propiciar a los alumnos un primer evento de alfabetización académica) y las estrategias de enseñanza empleadas, especialmente, el análisis de la estructura y las características del artículo en un periódico específico de la disciplina. Por todo ello, se puede pensar que el hecho de que la materia de María sea inicial haga que ponga más énfasis en lo que Lea y Street (2006) llaman estudio de habilidades y que por eso hay mayor cantidad de comentarios dirigidos a los estratos más bajos. Sin embargo, como vimos, no se ha dejado de lado la atención al contexto y al género, sino que esas dimensiones han sido consideradas desde la lectura y el análisis de los textos de la bibliografía como ejemplares representativos de los géneros de la disciplina. En ese punto, puede empezar a esbozarse una explicación de las diferencias encontradas en la comparación de los comentarios de ambas docentes. Para desarrollarla, nos detendremos, a continuación, en los datos provistos por Mariana.

Para comenzar, es preciso recordar que, según la misma Mariana explicita, su punto de partida para atender a la escritura y a la alfabetización académica se relaciona con lo que ella reconoce como una falta de atención pedagógica en Ciencias Sociales con respecto a las metodologías necesarias a la escritura en esta área del conocimiento: la docente evalúa la formación metodológica en la producción de los textos relacionados con la producción de conocimientos en 
Ciencias Sociales como una carencia en la educación del nivel superior.

Para fundamentar su posición, Mariana plantea que en otras áreas, como las Ciencias Exactas y Naturales, los estudiantes comienzan a participar de investigaciones y producciones colectivas desde los inicios de sus carreras. Y considera que resulta necesario atender a esa cuestión. En ese sentido, pone de manifiesto que, desde su punto de vista, cada cultura disciplinar adhiere a distintas conformaciones internas, lo que puede deberse a distintas visiones ideológicas sobre la producción de conocimiento.

Desde otro ángulo, notamos que tanto los comentarios sobre las producciones de sus estudiantes como las ideas que Mariana expresa sobre la alfabetización académica se vinculan con los objetivos de la asignatura. En efecto, teniendo en cuenta que sus alumnos están próximos a la graduación, ella busca formarlos en una práctica social corriente en el campo profesional en que se insertarán: la evaluación de programas sociales mediante herramientas teórico-analíticas. Así, Mariana adhiere, implícitamente, a una concepción de la escritura como una práctica social, con una visión genérica incluida, y a un modelo de alfabetización que se corresponde con el de socialización académica y disciplinar. Coincide, en este punto, con ideas teóricas del campo de la cultura escrita que señalan que la participación de los sujetos en las distintas culturas disciplinares depende, en gran medida, de la apropiación de los géneros (WERTSCH, 1991), por lo que la formación (el "entrenamiento" y la "preparación", en palabras de Mariana) de los nuevos miembros de las comunidades requiere, necesariamente, de la instrucción sobre las características de los distintos géneros que en ellas se emplean.

Los estudios de casos ahora presentados y analizados procuraron hallar respuestas a nuestras interrogantes de investigación. Se intentó saber, primero, si las concepciones sobre la escritura y las epistemologías disciplinares de las docentes varían según el área del conocimiento en que María y Mariana se inscriben: lingüística histórica y políticas sociales, respectivamente. En principio, observamos que ambas adhieren a una concepción de la escritura como proceso. En ese sentido, el área de conocimiento no parece tener influencia en la adopción de una visión sobre la escritura. Sin embargo, se encontró que Mariana hace también hincapié en aspectos epistemológicos y metodológicos involucrados en la escritura propia de los procesos de investigación y en el desarrollo de los razonamientos propios de las Ciencias Sociales.

Esas observaciones se ligan, a nuestro juicio, con los objetivos de la asignatura 
que cada una dicta y, también, con la ubicación de la materia en la malla curricular. Así, los estudiantes de Mariana se ven más exigidos en alcanzar formas avanzadas de la escritura porque ya están a punto de concluir sus estudios de grado y prontos a insertarse en el campo de las políticas sociales. Los de María, por su parte, recién comienzan a transitar los estudios superiores y se encuentran en una etapa de exploración de los géneros y las convenciones de la disciplina.

Por otro lado, buscamos dilucidar si el foco de atención de los comentarios se relaciona con un tipo de alfabetización académica delineada por el profesor y de qué manera se plantea tal relación. El análisis de los comentarios mostró una diferencia significativa entre las docentes: mientras María enfatizó el estrato notacional, Mariana hizo hincapié en el estrato discursivo. Estos datos, leídos de manera aislada, podrían sugerir que las profesoras se encuadran en modelos muy diferentes de alfabetización académica y que la primera solo buscaría que los alumnos conozcan y apliquen normas. Sin embargo, la interpretación de estos resultados cambia si se los lee a la luz de las informaciones originadas en las entrevistas.

En efecto, en los encuentros personales cada una de las profesoras mostró tener conciencia de que las necesidades de los estudiantes eran diferentes, sobre todo por su grado de avance en los estudios y, consecuentemente, han alcanzado distintos grados de dominio de la escritura académica. A la vez, se observó que en asignaturas iniciales como la de María los procesos de enseñanza de la escritura también se desarrollan en instancias más ligadas a la lectura, específicamente, a un tipo de lectura que se detiene en señalar aspectos genéricos y discursivos propios de una comunidad disciplinar. En cambio, en asignaturas de finales de carrera se pone el énfasis en la producción de géneros avanzados y en el desarrollo de una voz propia. Se estimula, así, un tipo de alfabetización focalizada en el contexto profesional y en los géneros avanzados.

De acuerdo con ese análisis, parece que ambas docentes contemplan distintas dimensiones de la escritura: como práctica social específica de una cultura disciplinar, como género de esa comunidad y como un proceso que requiere de la atención de los profesores durante la etapa de formación. En cuanto al modelo de alfabetización, surge que el predominante en los casos es el de socialización académica, en tanto, se prioriza el manejo de los géneros propios y el conocimiento de las prácticas sociales de los campos del saber en las cuales se inscriben las asignaturas. 
Para finalizar, queremos llamar la atención sobre la metodología adoptada. En primer lugar, no podemos dejar de señalar que el estudio es una primera exploración de un corpus acotado, por lo que resulta limitado y requiere una profundización y una ampliación de la muestra. Dicho esto, encontramos que la complementación del análisis de los comentarios con las entrevistas ha resultado más que provechoso y ha permitido comprender con mayor profundidad las motivaciones y los procedimientos que las dos docentes suelen adoptan para implementar la alfabetización académica en sus cursos.

\section{Implicaciones pedagógicas}

Para cerrar, queremos plantear algunas implicaciones pedagógicas surgidas de nuestro estudio. De los resultados alcanzados, dos llamaron la atención. En primer lugar, ambas docentes muestran adhesión a una concepción de la escritura que contempla tanto los procesos como los géneros y el contexto social y epistemológico de la asignatura; en segundo término, las dos dan cuenta de una concepción del aprendizaje interesada en las necesidades de los estudiantes. Sin embargo, hemos observado que el foco de sus comentarios cambia. Como ya hemos señalado, esta diferencia puede deberse a la ubicación de sus asignaturas en los planes de estudio. Teniendo en cuenta que los estudiantes de las asignaturas iniciales desconocen las normas propias del mundo académico, los comentarios de la docente se dirigen a explicitarlas. En cambio, hacia el final de la carrera, importa más promover ajustes en el plano del discurso para responder a los requisitos del mundo profesional.

De esos resultados surge, como implicación para el desarrollo de programas integrales de escritura académica, la necesidad de abarcar los diversos planos involucrados en la producción escrita, desde los contextuales hasta los más básicos de la expresión lingüística. Así, la enseñanza de la escritura académica puede empezar por el conocimiento de las normas de la disciplina y por la lectura de los géneros más típicos, como se observa en el diseño seguido por María. En las materias finales, como la de Mariana, es preciso enfocarse en cuestiones discursivas, genéricas y contextuales, de modo de facilitar el tránsito de los estudiantes al campo de actuación profesional. En todos los casos, se impone tener en cuenta las necesidades de los estudiantes. 
De acuerdo con lo anterior, resulta imprescindible investigar cuáles son las necesidades de aprendizaje de la escritura de los estudiantes y cómo cambian a lo largo de la carrera. Incluso, es importante diseñar programas que contemplen un abordaje sistemático de la alfabetización académica, de modo a poder abarcar todas las dimensiones involucradas en ese proceso.

\title{
Lectures writing and academic literacy conceptions: a contrastive study
}

\begin{abstract}
This paper present a contrastive and explanatory study about writing and academic literacy conceptions created and reproduced by written feedback to texts of students of tertiary education made in form of added comments on the same texts. On the basis of intentional selection of two cases, the paper focuses on the examination of which aspects two lectures draws attention to on their remarks. The first is from Brazil and is teacher of an initial subject of a undergraduate course and the other is from Argentina and is teacher of a final subject of another undergraduate course. Written feedback data is supported by complementary data collected from semistructured interviews to those teachers. The analysis presented along this paper contemplates the different speeches about writing (IVANIČ 2004) and models of literacy (LEA; STREET 1998). While comments analysis alone allows to believe they departure for different writing and academic literacy conceptions, the same data seen in the context described in the interviews reveals that, in fact, there are common believes and that the divergences founded are due to discrete subject aims and to the peculiar needs of each class of students.
\end{abstract}

Keywords: Writing. Academic Literacy. Written feedback. Conceptions. Lecturers.

\section{Referências}

ANSON, Chris. M. Response styles and ways of knowing. In ANSON, Chris M. (Ed.) Writing and response: Theory, practice, and research. Illinois: National Council of Teachers of English - Urbana, p. 332-66, 1989 
BAJTIN, Mijaíl. El problema de los géneros discursivos. In: BAJTIN, Mijaíl. Estética de la creación verbal. Buenos Aires: Siglo XXI, p.248-93, 1979.

BAZERMAN, Charles; LITTLE Joseph; CHAVKIN Teri et al. Writing Across the Curriculum. Reference guides to rhetoric and composition. West Lafayette, E.E.U.Y.: Parlor Press / WAC Clearinghouse, 2005.

BERKENKOTTER. Carol; HUCKIN, Thomas. Genre Knowledge in Disciplinary Communication: Cognition/Culture/Power. Mahwah, N. J. / E.E.U.U.: Lawrence Erlbaum, 1995.

BOURDIEU, Pierre; de SAINT MARTIN, Monique. Les catégories de l'entendement professoral. In: Actes de la recherche en sciences sociales, v.1(3), p.68-93, may. 1975.

BRASLAVSKY, Berta. ¿Primeras letras o primeras lecturas?: una introducción a la alfabetización temprana. Buenos Aires: Fondo de Cultura Económica, 2004.

CARLINO, Paula. Alfabetización académica: un cambio necesario, algunas alternativas posibles. Educere, v. 6, n. 20 (2003), p. 409-20, ene./feb./ mar. 2003. CARLINO, Paula. Alfabetización académica diez años después. Revista mexicana de investigación educativa, v. 18, n. 57, p. 355-381, abr./jun. 2013.

PARODI, GIOVANNI. Géneros académicos y géneros profesionales: Accesos discursivos para saber y hacer. Valparaíso: Pontificia Universidad Católica de Valparaíso, 2008.

COFFIN, Caroline; CURRY, Mary Jane; GOODMAN, Sharon et al. (2005). Teaching academic writing: A toolkit for higher education. Londres, Nova Yorque: Routledge, 2005.

COPE, Bill ; KALANTZIS, Mary. The Powers of Literacy: a genre approach to teaching literacy. Londres: Falmer, 1993.

Di BENEDETTO, Silvia; CARLINO, Paula (2007). Correcciones a exámenes escritos en la universidad: cómo son y para qué sirven a los alumnos. In: Memorias de las XIV JORNADAS DE INVESTIGACIÓN EN PSICOLOGÍA Y TERCER ENCUENTRO DE INVESTIGADORES EN PSICOLOGÍA DEL MERCOSUR, tomo I, Buenos Aires: Facultad de Psicología-Universidad de Buenos Aires, p. 273275. Disponible en $<$ https://sites.google.com/site/jornadasgiceolem/universidad. $>$ Acceso en: 12 oct. 2016.

FERRIS, Dana. R. Response to student writing: Implications for second language students. Mahwah, N. J. E.E.U.U.: Lawrence Erlbaum, 2003. 
FLOWER, Linda. y HAYES, John R. A cognitive process theory of writing. College composition and communication, v. 32, n. 4, p.365-87, dic. 1981.

HYLAND, Ken. Teaching and researching writing. Londres: Longman, 2002.

HYLAND, Ken. Genre-based pedagogies: A social response to process. Journal of second language writing, v. 12, n. 1, p. 17-29, dic. 2003.

HYLAND, Ken. Disciplinary Discourses. Social Interactions in Academic Writing. Ann Arbor: University of Michigan Press, 2004.

HYLAND, Ken; HYLAND, Fiona. Feedback on second language students' writing. Language Teaching, v. 39, n. 2, p. 83-101, abr. 2006 a.

HYLAND, Ken; HYLAND, Fiona. Feedback in Second Language Writing. Cambridge: Cambridge University Press, $2006 \mathrm{~b}$.

HYLAND, Ken; HYLAND, Fiona (2001). Sugaring the pill: praise and criticism in written feedback. Journal of Second Language Writing, v. 10, n. 3, p. 185212, ago. 2001.

HYON, Sunny. Genre in three traditions: Implications for ESL. TESOL quarterly, v. 30, n. 4, p. 693-722, dic.1996.

IVANIČ, Roz. Writing and identity: The discoursal construction of identity in academic writing. Amsterdã: John Benjamins, 1998.

IVANIČ, Roz. Discourses of writing and learning to write. Language and education v. 18 , n. 3, p. 220-245, março, 2004. Disponible en <http://www. tandfonline.com/toc/rlae20/18/3?nav=tocList $>$. Acceso en: 06 feb. 2017.

IVANIČ, Roz.; CLARK, Romy; RIMMERSHAW, Rachel. (2000). "What am I supposed to make of this?: the messages conveyed to students by tutors' written comments" . In: Lea, Mary. R., Stierer, Barry (orgs.). (2000) Student writing in higher education: New contexts. Buckingham: The Open University Press/ Society for Research into Higher Education, 2000, p. 47-65.

JEFFERY, Francie; SELTING, Bonita. Reading the Invisible Ink: Assessing the Responses of Non-Composition Faculty. Assessing Writing, v. 6, n. 2, p. 179-197, 1999.

KRESS, Gunther; van LEEUWEN, Theo. Reading images: The grammar of visual design. New York: Routledge, 1996.

KUMAR, Vijay; STRACKE, Elke. Examiners' reports on theses: feedback or assessment? Journal of English for Academic Purposes, v. 10, p.211-222, dic. 2011. 
LEA, Mary R.; STREET, Brian V. Student writing in higher education: An academic literacies approach. Studies in higher education, v. 23, n. 2, p.157-72, jun. 1998.

LEA, Mary R.; STREET, Brian V. The "academic literacies" model: Theory and applications. Theory into practice, v. 45, n. 4, p. 368-77, nov. 2006.

LILLIS, Theresa. Student writing as "academic literacies": Drawing on Bakhtin to move from critique to design. Language and education, v. 17, n. 3, p. 192-207, 2003.

LILLIS, Theresa; SCOTT, Mary (2007). Defining academic literacies research: Issues of epistemology, ideology and strategy. Journal of applied linguistics. V. 4, n. 1, p. 5-32, dic. 2007.

MARTIN, James Robert. English text: System and structure. Amsterdam: John Benjamins, 1992.

MARTIN, James Robert y ROSE, David. Genre relations: Mapping culture. London: Equinox, 2008.

NATALE, Lucía Antonia. (Coord.) (2013a). El semillero de la escritura. Las tareas escritas a lo largo de tres carreras de la UNGS. Los Polvorines: UNGS. Disponible en $<$ http://www.ungs.edu.ar/areas/publicaciones/582/el-semillero-dela- escritura.html>. Acceso en 02 feb. 2017.

NATALE, Lucía Antonia. Integración de enfoques en un programa institucional para el desarrollo de la escritura académica y profesional. Revista Mexicana de Investigación Educativa. v. 18, n. 58, p. 685-707, jul-sept. 2013b.

NATALE, Lucía Antonia. Relaciones intersubjetivas entre expertos y novatos en devoluciones escritas de docentes universitarios. Legenda, v.17, n. 16, p. 86-114, $2013 \mathrm{c}$.

NATALE, Lucía Antonia ; STAGNARO, Daniela; PÉREZ, Inés ; RÍOS, Leticia. (2017). El Programa de Desarrollo de Habilidades de Lectura y Escritura a lo largo de la Carrera, de la Universidad Nacional de General Sarmiento (Argentina). In: NATALE, Lucía Antonia y STAGNARO, Daniela (orgs.). Alfabetización académica: un camino para la inclusión en el nivel superior. Los Polvorines: UNGS, p.161-199, 2017.

NESI, Hillary; GARDNER, Sheena. Genres across the disciplines: Student writing in higher education. Cambridge: Cambridge University Press, 2012. 
SCARDAMALIA, Marlene y BEREITER, Carl. "Knowledge telling and knowledge transforming in written composition”. In : ROSENBERG, S. (Ed). Advances in applied psycholinguistics, Vol. 2: Reading, writing, and language learning, New York, NY, US: Cambridge University Press, p.142-176, 1987.

STREET, Brian. Literacy in theory and practice. Cambridge: Cambridge University Press, 1984.

SWALES, John. Research genres. Cambridge: Cambridge University Press, 2004.

SWALES, John. Genre analysis: English in academic and research settings. Cambridge: Cambridge University Press, 1990.

WERTSCH, James. Voices of the mind: A sociological approach to mediated action. Cambridge: Harvard University Press, 1991. 